\title{
10. NASA-LRC Faint Meteor Spectra
}

\author{
Gale A. Harvey \\ Langley Research Center, NASA \\ Hampton, Virginia
}

\begin{abstract}
$A$ brief description is given of the instrumentation, the facilities, and the patrol technique used by the NASA Langley Research Center Faint Meteor Spectra Patrol. A classification of 500 meteor spectra obtained in the first 2-1/2 years of the patrol is given. The general characteristics of "typical" spectra are discussed and preliminary conclusions drawn. Examples of unusual spectra are briefly described.
\end{abstract}

$\mathrm{T}$

HE NASA LANGLEy RESEARCH CENTER (LRC) has established a meteor spectra patrol in the south-central part of New Mexico. This patrol, the NASA-LRC Faint Meteor Spectra Patrol, is operated by Smithsonian Astrophysical Observatory personnel under contract to LRC. The patrol direction, spectral data reduction, and analysis are performed at the Langley Research Center.

The primary spectrographs of the patrol are 15-cm aperture, f/1.3 Maksutov slitless spectrographs equipped with photomultiplier-actuated shutter systems. Twenty Maksutov spectrographs are assigned to the patrol. The first meteor spectrum was recorded on August 20, 1968. The patrol is now yielding spectra at the rate of several hundred per year.

The NASA-LRC Faint Meteor Spectra Patrol was initially established as part of the NASA meteoroid hazard research. The patrol was the primary data source for LRC meteor spectral studies. The primary objective of these studies was to relate the well determined luminous efficiency of low-velocity iron and nickel artificial meteors to natural meteors. However, by the time the patrol became operational, this had been done, on a statistical basis with the luminous efficiency study of iron and stone meteors by Cook, Jacchia, and McCrosky (1963), and the luminous-efficiency velocity-dependence study by Verniani (1967). At the same time, detailed understanding of meteor radiation processes was lacking, and the heterogeneous nature of the meteoroid environment was becoming more and more apparent (Grygar et al., 1968; Lindblad, 1963; Millman, 1967). Accordingly, the LRC meteor spectral studies were redirected toward study of meteor radiation and composition measurements.

The purpose of this paper is to describe briefly the NASA-LRC Faint Meteor Spectra Patrol and the first 500 spectra obtained by the patrol. Statistical conclusions are given and examples of meteor spectra are presented and discussed.

\section{INSTRUMENTATION AND PATROL TECHNIQUE}

The Langley Research Center developed fast, extended wavelength- range spectrographs (Harvey, 1967) to obtain spectra of the artificial meteors of the Meteor Simulation Program (Ayers et al., 1970). Subscquently, several models of Maksutov slitless spectrographs with high nearultraviolet transmission have been fabricated for the Faint Meteor Spectra Patrol. The primary spectrographs of the Faint Meteor Spec- 
tra Patrol are eleven 15-cm aperture, $f / 1.3$ spectrographs with first-order inverse dispersions of $165 \AA / \mathrm{mm}$, or $123 \AA / \mathrm{mm}$. Nine other Maksutov spectrographs of different designs and with inverse dispersions of 450 and $1300 \AA / \mathrm{mm}$ are also assigned to the patrol. The circular field of view of most of the spectrographs is $21^{\circ}$ in diameter.

Since very high-speed film (ASA 8000) is used in the spectrographs, continuous exposure of the spectrographs to the night sky requires a prohibitive amount of plate changing and film processing. To alleviate this problem, a photoelectric meteor detection-shutter system has been developed for the spectrographs. This shutter system effects a 2-s exposure when a meteor occurs in the field of view of the spectrograph. With the exception of an extreme sensitivity to lightning, the photoelectric shutter systems have proven efficient and well matched to the spectrographs.

Until December 1970, no ballistic data were available for sporadic meteors obtained by the patrol, and complete reductions were limited to major shower meteors. Since December 1970, a two-station ballistic system has been in operation, and it is expected that approximately 10 percent of the sporadic meteor spectra will have ballistic data after this time.

The Faint Meteor Spectra Patrol is operated on a routine nightly basis with highly skilled master observer Roy Proctor and observer Norbert Roth having alternate nights on. Special emphasis is placed on optimum operation during major meteor showers. The patrol is generally operated when cloud cover is less than 50 percent. A more complete description of the NASALRC Faint Meteor Spectra Patrol is given by Harvey (1971).

\section{STATISTICS OF SPECTRA}

Most of the spectra obtained by the Faint Meteor Spectra Patrol are in the brightness interval +1 to -3 absolute photographic meteor magnitude. This deduction was made by comparing these spectra with several absolutely reduced spectra, and from limited visual observations. The first meteor spectrum was obtained during the night of August 20, 1968. The 500th spectrum was obtained during the night of February 28, 1971. The most spectra obtained in one night, 26 , were obtained on the night of December 13, 1969. Of the first 500 spectra, 345 have 1 to 9 spectral features, 86 have 10 to 19 features, 51 have 20 to 49 features, and 18 have more than 49 features. These groups correspond to Millman's d, c, b, and a class spectra respectively (Millman, 1963), and are based on examination with a visual comparator.

Typical examples of " $\mathrm{d}$ " spectra are shown in figure 1. Figure 1 (a) is a reproduction of spectrum 374 and was recorded on an $f / 0.83,150-\mathrm{mm}$ aperture spectrograph of $500 \AA / \mathrm{mm}$ inverse dispersion. Figure $1(b)$ is a reproduction of spectrum 322 and was recorded on an $f / 1.3,150-\mathrm{mm}$ aperture spectrograph of $165 \AA / \mathrm{mm}$ inverse dispersion. This spectrograph was equipped with a rotating shutter which produced 20 occultations per second. The strongest features in these spectra are multiplets 4,5 , and 20 of neutral iron.

Typical examples of "c" spectra are shown in figure 2. Figure 2(a) is a reproduction of spectrum 454 and was recorded on an $f / 0.83,150-\mathrm{mm}$ aperture spectrograph of $500 \AA / \mathrm{mm}$ inverse dispersion. Figure 2(b) is a reproduction of spectrum 13 and was recorded on an $f / 1.3,150-\mathrm{mm}$ aperture spectrograph of $165 \AA / \mathrm{mm}$ inverse dispersion. Again, the strongest features in the spectra are multiplets 4,5 , and 20 of iron.

Typical examples of " $b$ " spectra are shown in figure 3 . Figure $3(\mathrm{a})$ is a reproduction of spectrum 244 and was recorded on an $f / 1.3,150-\mathrm{mm}$ aperture spectrograph of $165 \AA / \mathrm{mm}$ inverse dispersion. Although the green magnesium triplet and the sodium $D$ lines are quite strong in this spectrum, multiplets 4,5 , and 20 are still the strongest features in the spectrum. Figure $3(\mathrm{~b})$ is a reproduction of spectrum 246 and was recorded on an $f / 1.3,150-\mathrm{mm}$ aperture spectrograph of $123 \AA / \mathrm{mm}$ inverse dispersion. Fortythree features were counted by visual examination; however, a microdensitometer tracing would easily allow more than 50 features to be identified. Although the sodium $D$ lines are strong, multiplets, 4,5 , and 20 of iron are again the strongest features.

Typical examples of " $a$ " spectra are shown in figure 4. Figure $4(a)$ is a reproduction of spec- 

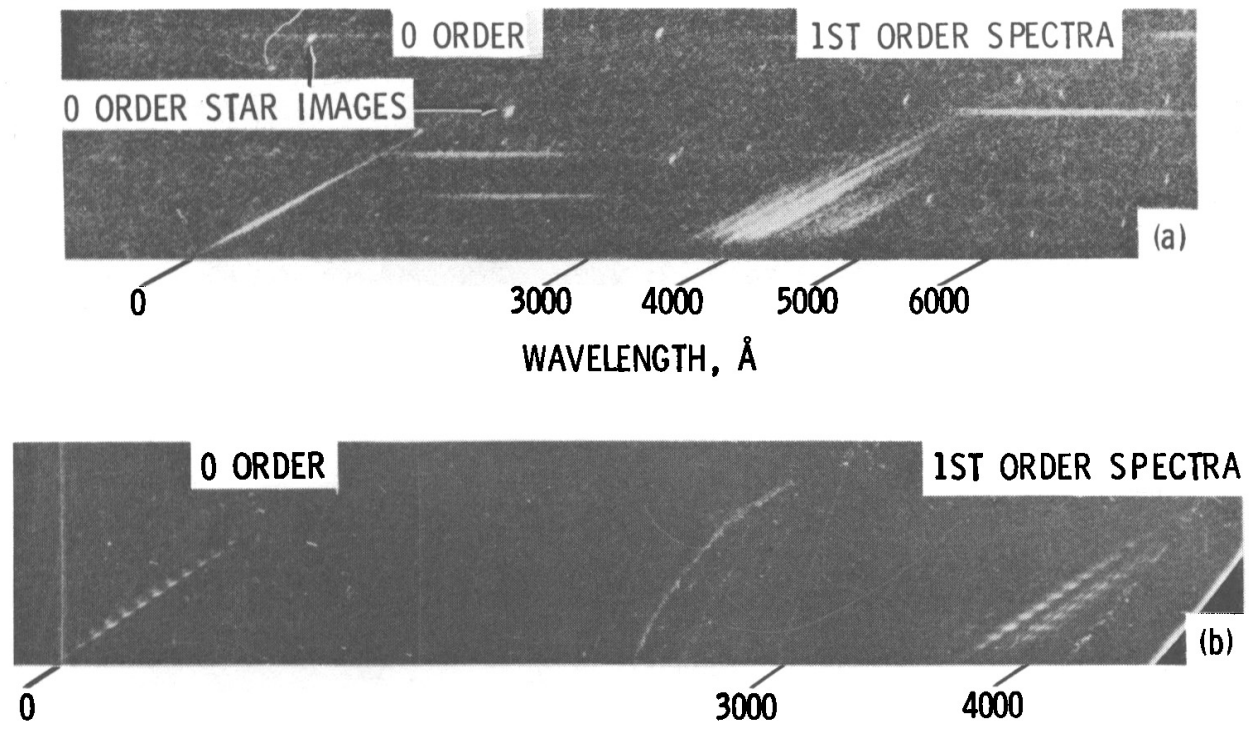

Figure 1.-Meteor spectra with one to nine features ("d" spectra). (a) Spectrum No. 374. (b) Spectrum No. 322 .
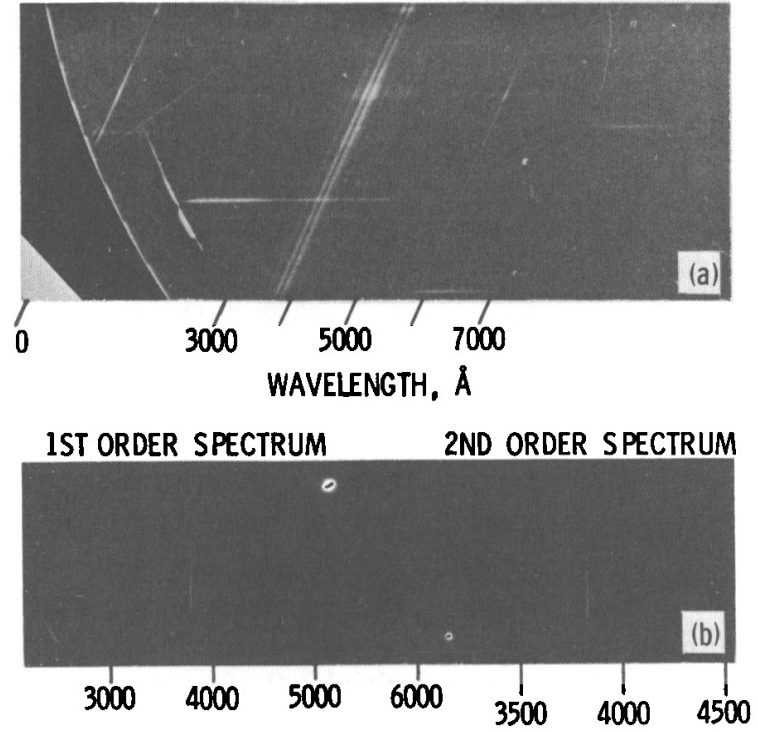

Figure 2.-Meteor spectra with 10 to 19 features ("c" spectra). (a) Spectrum No. 454. (b) Spectrum No. 13.

trum 166 and was recorded on an $f / 1.3,150-\mathrm{mm}$ aperture spectrograph of $123 \AA / \mathrm{mm}$ inverse dis persion. This spectrum is of a Geminid meteor and has been analyzed in a companion paper (Harvey, 1972). Multiplets 4, 5, and 20 of iron are again the strongest features in the spectrum. Figure $4(\mathrm{~b})$ is a reproduction of spectrum 67 (a) and was recorded on an $f / 1.3,150$-mm aperture spectrograph of $123 \AA / \mathrm{mm}$ inverse dispersion. This spectrum is of a Perseid meteor. The strongest lines are from multiplet 1 of ionized calcium, multiplets 4,5 , and 20 of iron, and the sodium $D$ lines.

In figures 1 to 4 , multiplets 4,5 , and 20 of neutral iron are the dominant radiation in most of the spectra. Of the first 500 meteor spectra from the Faint Meteor Spectra Patrol, approximately 60 percent have multiplets 4,5 , and 20 of iron as the dominant radiation and hence are Millman's (1963) type $Z$ spectra. Twenty-five percent of the spectra have magnesium or sodium as the strongest radiation and thus are type $X$ spectra, while 8 percent have neutral calcium or oxygen as the strongest radiation, and 7 percent are of indeterminant class. Only about 2 percent of the spectra have the $H$ and $K$ lines of calcium as the strongest radiation and hence are type $Y$ spectra. One to 2 percent of the first group are nearly pure iron spectra, expected of nickel-iron type meteoroids. As will be discussed later, a small percentage of the second group are essentially iron-free spectra. Except for these iron-rich and iron-poor spectra, both of the first two groups are generally consistent with compositions similar to typical stony meteorites. 


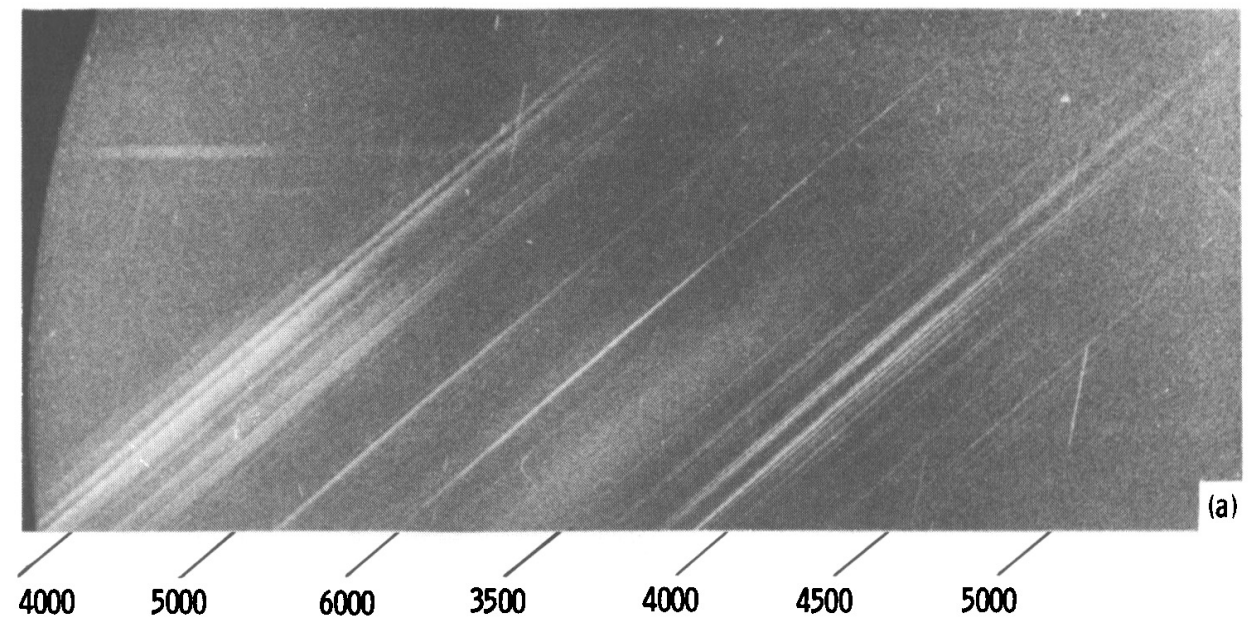

WAVELENGTH, $\AA$

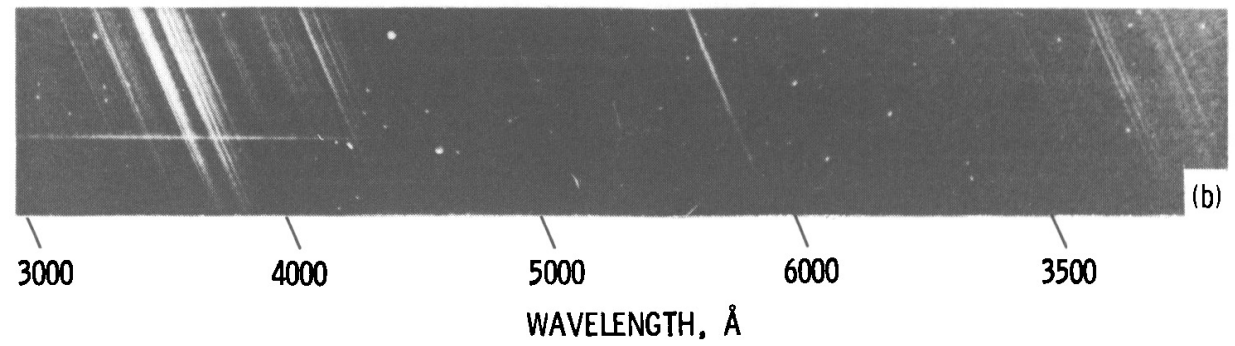

Figure 3.-Meteor spectra with 20 to 49 features ("b" spectra). (a) Spectrum No. 244. (b) Spectrum No. 246.

These statistics of meteor radiation are important observational results, because they offer strong support for the most vulnerable assumptions used in the optical meteor mass determinations. These assumptions are that the iron in slow metrors is the dominant radiator in the SuperSchmidt blue region of the spectrum, and that most meteors are of composition similar to typical stony meteorites. Thus, the photometric meteor mass determinations are statistically valid. However, this does not mean that all individual photometric meteor mass determinations are accurate.

\section{NONREPRESENTATIVE SPECTRA}

As scen in the previous section, iron is the dominant radiation species in most faint meteor spectra. However, iron deficient spectra have been obtained. Examples of extremely iron- deficient or iron-free meteors are shown in figure 5. Figure 5(a) is reproduced from spectrum 401 and was recorded on an $f / 1.3,150-\mathrm{mm}$ aperture spectrograph of $123 \AA / \mathrm{mm}$ inverse dispersion. Figure 5(b) is reproduced from spectrum 299 and was recorded on a similar spectrograph. The strongest feature in these spectra is the multiplet 3 of neutral magnesium. The other strong lines are multiplet 1 of ionized calcium, multiplet 2 of neutral calcium, and multiplet 2 of neutral magnesium. Multiplets 4 and 5 of iron are present, but very weak. Two similar speetra were among 39 prismatic spectra obtained from SuperSchmidt spectral patrols in 1966 to 1968. These were two of the better spectra, and at that time it was thought that this type of meteor might be fairly common. However, on the basis of classification of 500 spectra, only about 1 percent of all meteors have this type of spectrum.

A similar type of spectrum is shown in figure 

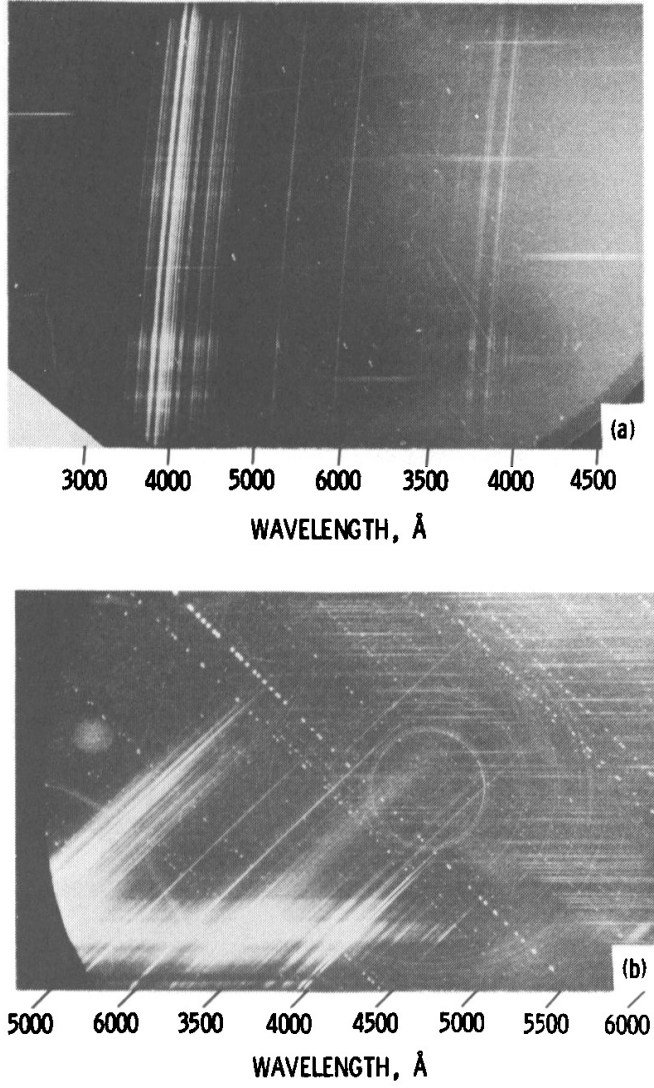

Figure 4.-Meteor spectra with more than 49 features ("a" spectra). (a) Spectrum No. 166. (b) Spectrum No. 67a.
6. Figure 6(a) is reproduced from spectrum 228 and figure $6(\mathrm{~b})$ is reproduced from spectrum 95 . Both are $123 \AA / \mathrm{mm}$ inverse dispersion spectra. They are two-line spectra with multiplet 3 of neutral magnesium being strong and multiplet 2 of neutral calcium being weak. They appear to be slow meteors of similar composition to the meteors of $5(\mathrm{a})$ and $5(\mathrm{~b})$. About 1 percent of the spectra are of this type.

On several meteor spectra, what appears to be a persistent feature near $3840 \AA$ has been observed. It is shown in figure 7. This radiation is present at the bottom of the first-order spectrum in figure 7 (a) and most strongly at the top of the second-order spectrum in figure $7(b)$. The abrupt beginning of the meteor is due to the opening of the shutter. The persistence of the $3840 \AA$ feature is denoted by its higher position along the trailed spectrum. This radiation appears to be present in several percent of the spectra. It is suspected that this feature is band radiation because it seems to occur in low dispersion spectra more often than in high dispersion spectra. It seems that in many meteor spectra, this is the strongest initial feature which fades as the usual iron and magnesium lines intensify. This radiation is difficult to analyze because it is in the region of multiplets 4 and 20 of neutral iron and 3 of neutral magnesium. Halliday

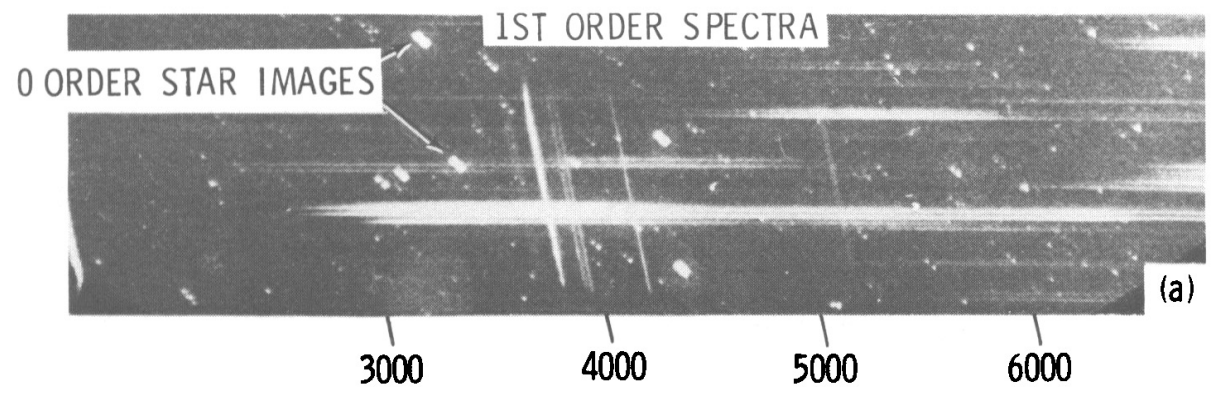

WAVELENGTH, $\AA$

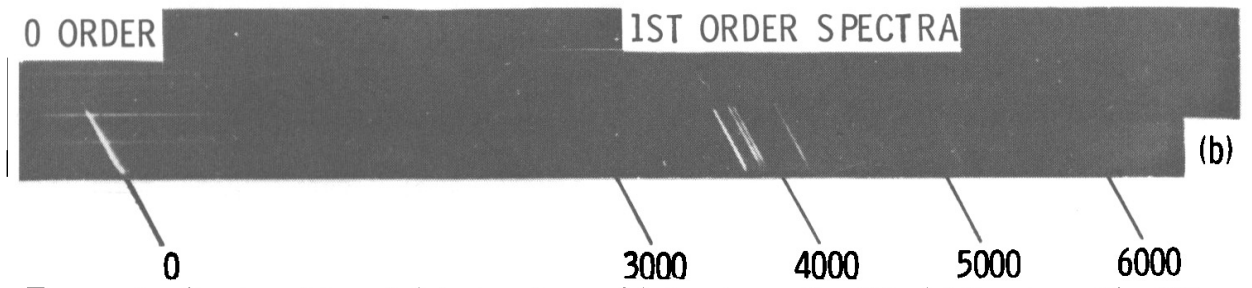

Figure 5.-Spectra of iron-deficient meteors. (a) Spectrum No. 401. (b) Spectrum No. 299. 

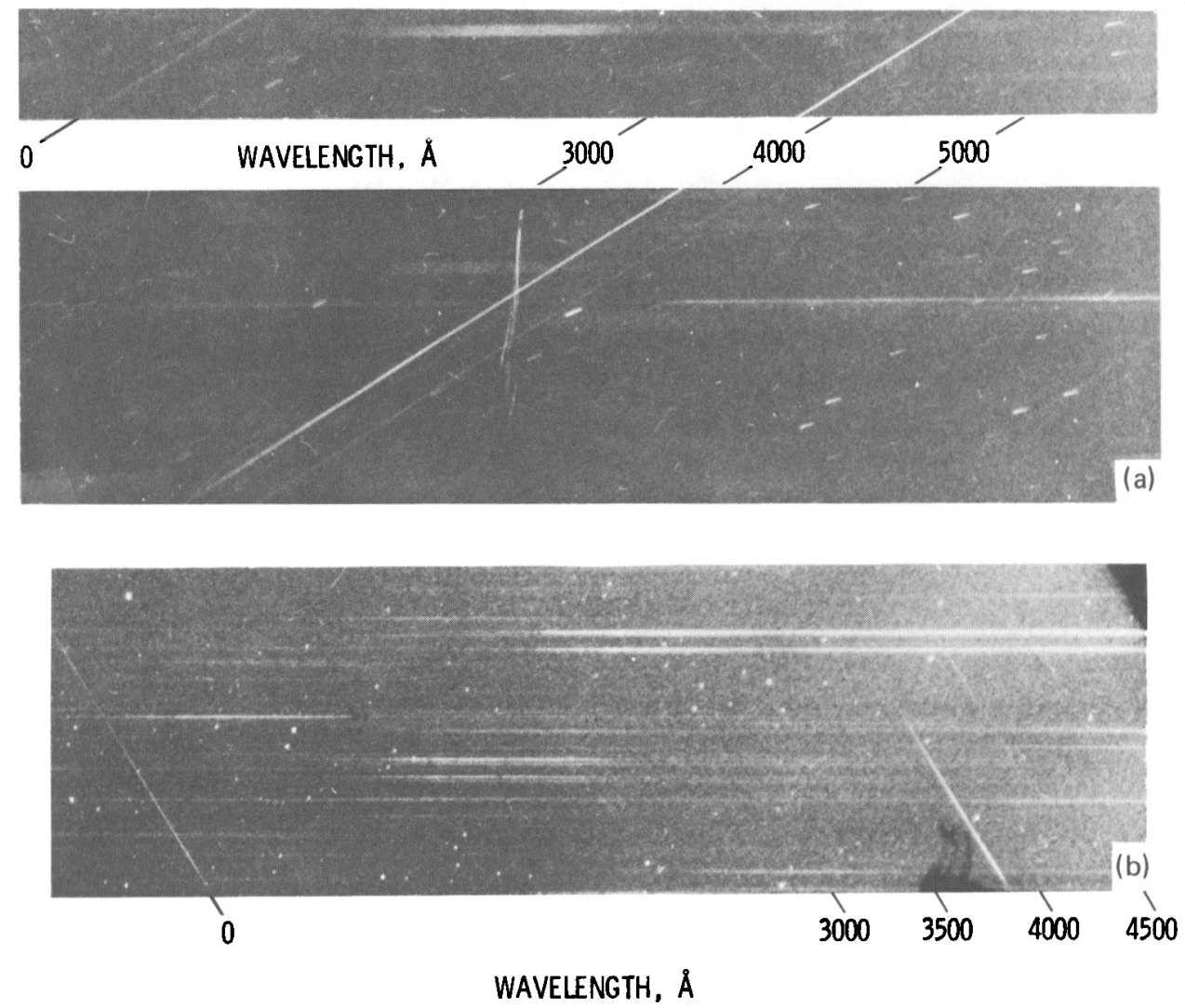

Figure 6.-Spectra of iron-deficient meteors. (a) Spectrum 228. (b) Spectrum No. 95.

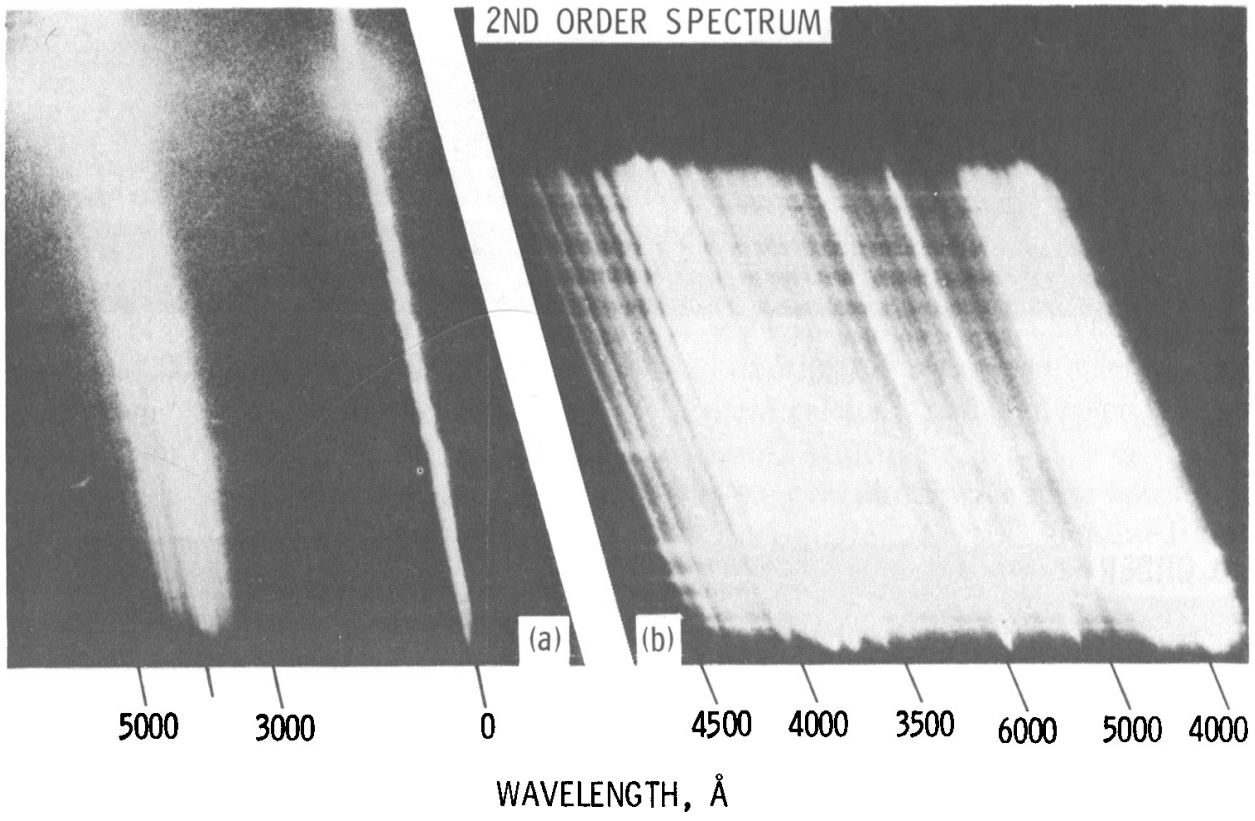

FIGURE 7.-Meteor spectra with persistent UV radiation. (a) Spectrum No. 24. (b) Spectrum No. 251. 


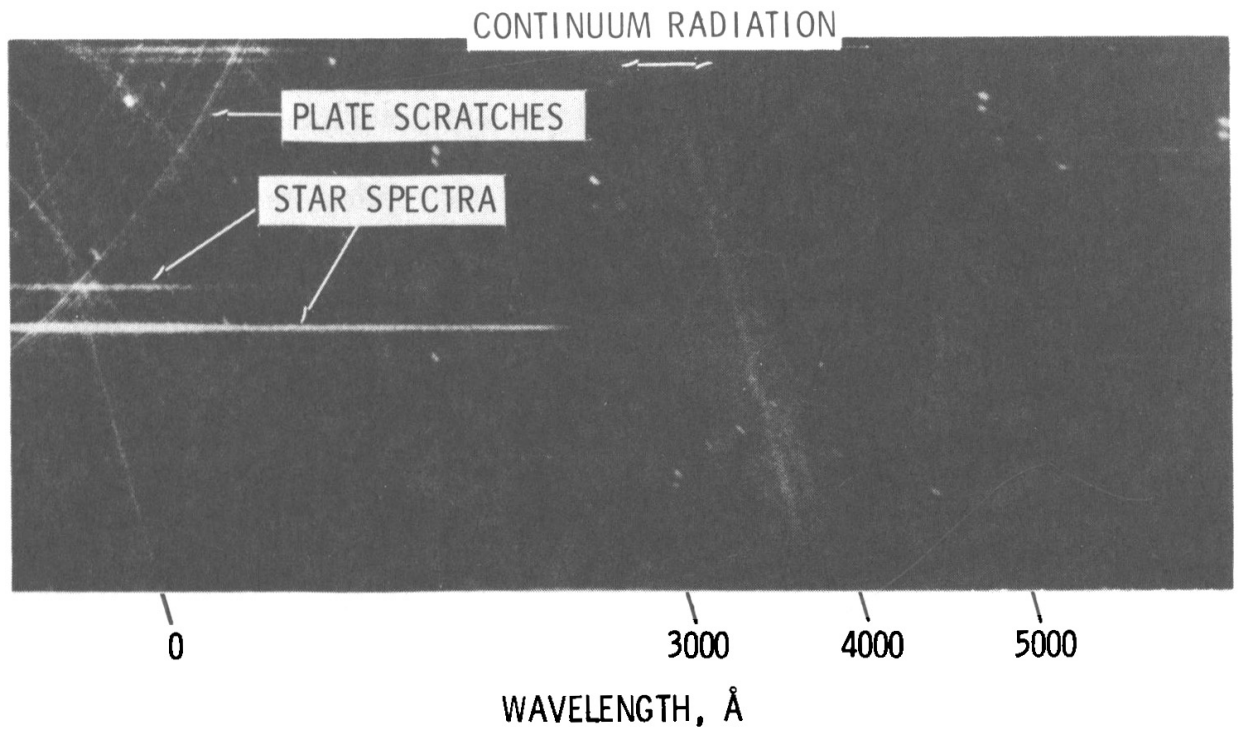

Figure 8.-Spectrum of faint, continuum radiation meteor (Spectrum No. 6).
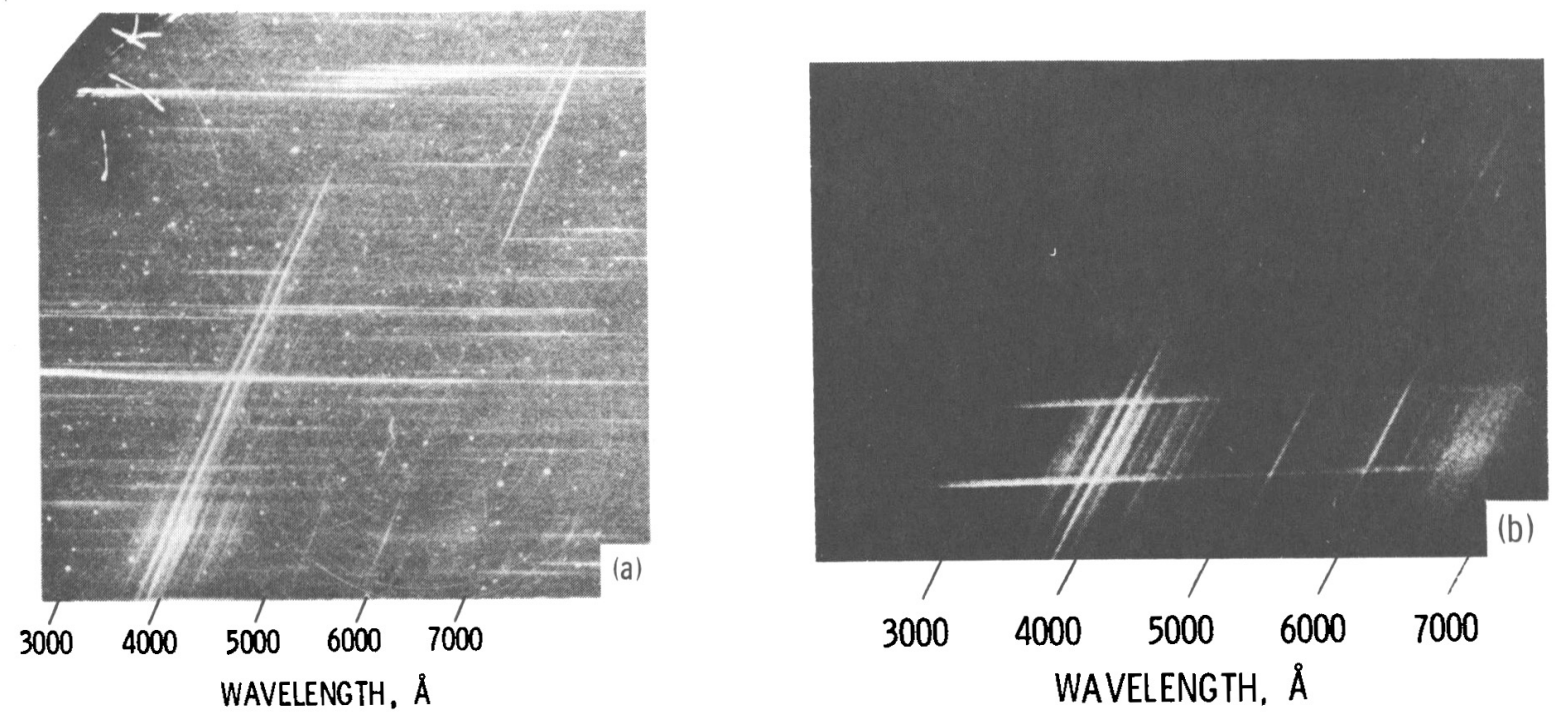

Figure 9.-Spectra of faint Perseid and Leonid meteors. (a) Spectrum No. 56, Perseid meteor.

(b) Spectrum No. 8, Leonid meteor.

(1969) discusses a similar, if not the same, feature in his UV Lyrid spectrum.

Almost all of the radiation of the 500 meteors recorded by the patrol is atomic line radiation. The most general exception is a low level of unresolved radiation in the $6000 \AA$ to $7000 \AA$ region. However, one meteor was recorded in which no atomic line radiation appears. This spectrum is shown in figure 8 . This figure is reproduced from spectrum 6 and was recorded on an $f / 0.83,150-\mathrm{mm}$ aperture spectrograph of $500 \AA / \mathrm{mm}$ inverse dispersion. The faint continuum extends from $3600 \AA$ to $4000 \AA$, with a stronger feature near $3800 \AA$. This is the only spectrum of this type obtained by the patrol to date. 
Fast, bright meteors are characterized by extreme dominance of the radiation from multiplet 1 of ionized calcium, multiplet 4 of ionized magnesium, and multiplet 2 of ionized silicon. Figure 9 shows spectra of faint Perseid and Leonid meteors in which the ionic radiation is absent, or at low levels relative to the neutral spectrum. The low strength of ionic radiation relative to neutral radiation, and the virtual absence of other multiplets of ionized calcium, magnesium, and silicon confirm that the observed ionic radiation in bright, fast meteors is anomalous.

Sodium abundances may be indicative of lifetimes and origins of meteoroids. The two spectra shown in figure 10 are indicative of the range of sodium abundances in meteoroids. Figure 10(a) is reproduced from spectrum 430 . Figure 10(b) is reproduced from spectrum 432 . The spectra were obtained on the same $f / 1.3,150-\mathrm{mm}$ aperture spectrograph of $123 \AA / \mathrm{mm}$ inverse dispersion on consecutive nights. In figure 10 (a) sodium is the dominant radiating element, in figure 10 (b) no sodium radiation is recorded. Both spectra have more than 50 features as determined from an examination with a visual comparator.

Preliminary conclusions which can be drawn from the NASA-LRC Faint Meteor Spectra are:

(1) Approximately 60 percent of optical meteors have similar spectra in which multiplets 4,5 , and 20 of iron are the dominant radiation. Thus, the optical meteor mass determinations, that are based on the assumption that the predominant radiation from slow meteors in the blue region of the spectrum is iron radiation, are statistically valid.

(2) A small percentage of meteoroids are essentially iron free.

(3) Persistent radiation near $3840 \AA$ occurs in faint meteors.

(4) $\mathrm{A}$ few ( $<0.5$ percent) faint meteors are not predominantly atomic line radiators.

(5) Ionic radiation in bright, fast meteors is anomalous.

(6) Sodium abundances in meteors vary widely.

\section{ACKNOWLEDGMENTS}

The author wishes to acknowledge the contribution of the patrol personnel; Roy Proctor, master observer and station manager, and Norbert Roth, observer. Their skill and dedication in implementing and operating a new observing system in a different mode of operation are responsible in large measure for the results of the subject patrol.
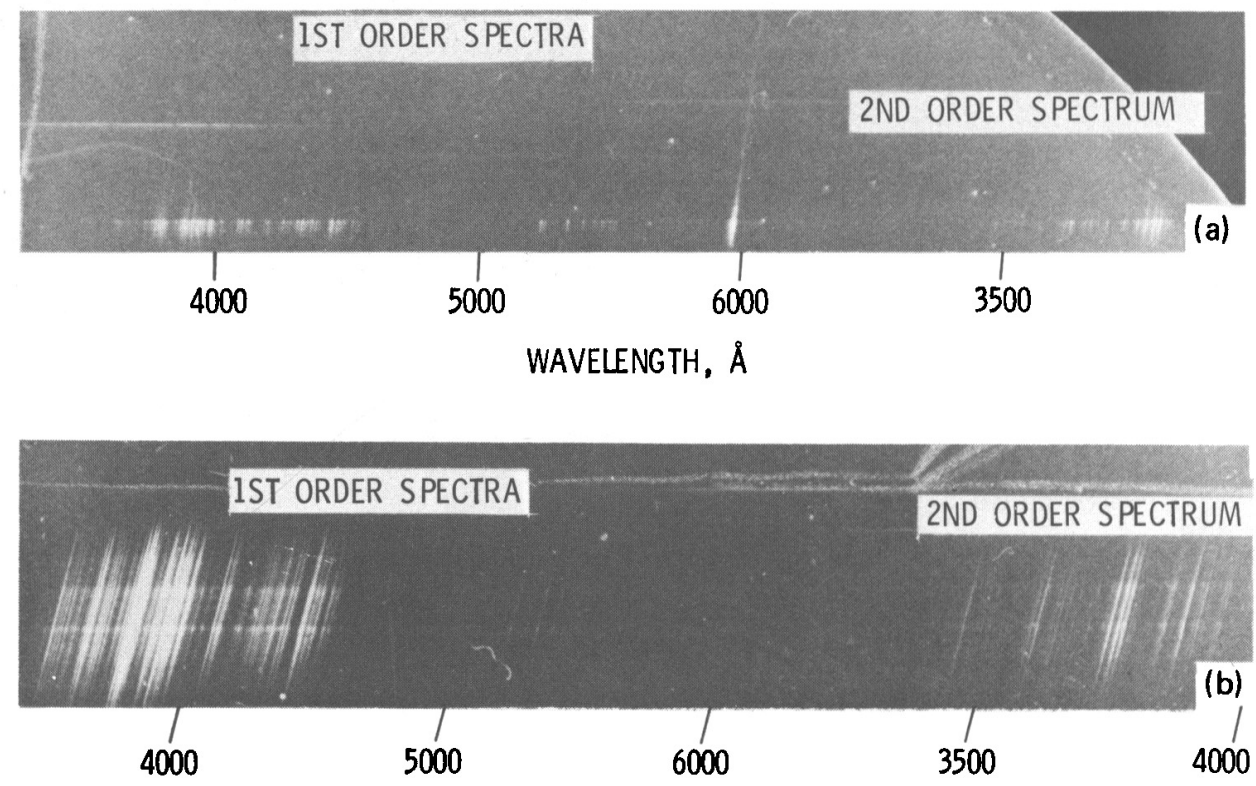

FIGURE 10.-Spectra of sodium rich and sodium-deficient meteors. (a) Spectrum No. 430, sodiumrich meteor. (b) Spectrum No. 432, sodium-deficient meteor. 


\section{REFERENCES}

Ayers, W. G., McCrosky, R. E., and Shao, C. -Y., 1970. Photographic observations of 10 artificial meteors, Smithson. Astrophys. Obs. Spec. Rept., No. 317, 1-40.

Cook, A. F., JacchiA, L. G., AND McCrosky, R. E., 1963. Luminous efficiency of iron and stone asteroidal meteors, Smithson. Contrib. Astrophys., 7, 209-220.

Grygar, J., Kohoutek, L., ANd Plavcoví, Z., 1968. Simultaneous radar and optical observations of meteors at Ondřejov in 1962, in Physics and Dynamics of Meteors, edited by $\check{\mathrm{L}}$. Kresák and P. M. Millman, D. Reidel Publ. Co., Dordrecht, Holland, 63-69.

Halliday, I., 1969. A study of ultraviolet meteor spectra, Publ. Dominion Obs., 25, 315-322.

Harvey, G. A., 1967. A description of four fast slitless spectrographs, NASA Tech. Note D-4145, 1-38.

- - 1971. The NASA LRC faint meteor spectra patrol, NASA Tech. Note D-6298, 1-25.

- , 1972. Spectral analysis of four meteors, see Paper No. 9, this volume.

LiNDBLAD, B. A., 1963. The relation between visual magnitudes and the durations of radar echoes, Smithson. Contrib. Astrophys, , 7, 27-39.

Millman, P. M., 1963. A general survey of meteor spectra, Smithson. Contrib. Astrophys., 7, 119-127.

- 1967. Some characteristics of the major meteor showers, Smithson. Contrib. Astrophys., 11, 105-108.

Verniani, F., 1967. Meteor masses and luminosity, Smithson. Contrib. Astrophys., 10, 181-195. 